\title{
The ROSAT all-sky survey catalogue of optically bright late-type giants and supergiants ${ }^{\star}$
}

\author{
M. Hünsch ${ }^{1,2}$, J.H.M.M. Schmitt ${ }^{1}$, and W. Voges ${ }^{1}$ \\ 1 Max-Planck-Institut für Extraterrestrische Physik, Giessenbachstr., 85740 Garching, Germany \\ ${ }^{2}$ Hamburger Sternwarte, Gojenbergsweg 112, 21029 Hamburg, Germany
}

Received March 26; accepted May 5, 1997

\begin{abstract}
We present X-ray data for all late-type (A, F, $\mathrm{G}, \mathrm{K}, \mathrm{M}$ ) giants and supergiants (luminosity classes I to III-IV) listed in the Bright Star Catalogue that have been detected in the ROSAT all-sky survey. Altogether, our catalogue contains 450 entries of X-ray emitting evolved latetype stars, which corresponds to an average detection rate of about 11.7 percent. The selection of the sample stars, the data analysis, the criteria for an accepted match between star and X-ray source, and the determination of $\mathrm{X}$-ray fluxes are described.
\end{abstract}

Key words: stars: activity — stars: coronae — stars: late-type - X-rays: stars - catalogs

\section{Introduction}

Late-type stars are among the most frequent classes of X-ray sources. As suggested by the study of the Sun, their X-ray emission is believed to be thermal emission from hot $\left(10^{6} \mathrm{~K}\right)$ coronal plasma. Hundreds of late-type stars were already detected by the Einstein and EXOSAT Observatories (cf. Vaiana et al. 1981) with X-ray luminosities mostly ranging from about solar level (i.e., $10^{27} \mathrm{erg} \mathrm{s}^{-1}$ ) up to $\sim 10^{31} \mathrm{erg} \mathrm{s}^{-1}$. Recent ROSAT observations of a complete sample of the nearest stars (Schmitt et al. 1995; Schmitt 1997) have demonstrated that virtually all late-type main-sequence stars are X-ray sources. It is generally accepted that the existence of outer convection zones (starting at late A-types) as well as rotation are essential conditions for magnetically heated stellar coronae. A relation between $\mathrm{X}$-ray luminosity and rotation velocity was first established by Pallavicini et al. (1981), and gave

Send offprint requests to: M. Hünsch, e-mail: mhuensch@rosat.mpe-garching.mpg.de

* Catalogue only available at CDS via anonymous ftp to cdsarc.u-strasbg.fr (130.79.128.5) or via http://cdsweb.ustrasbg.fr/Abstract.html an incentive for numerous further studies in the context of the rotation-activity relationship. In connection with angular momentum evolution stellar X-ray emission can thus be regarded as a rough age-indicator.

Contrary to main-sequence stars, not all late-type giants and supergiants were found as X-ray sources. In particular, IUE and Einstein observations suggested that in certain regions of the Hertzsprung-Russell (HR) diagram late-type giants possess hot transitions regions and coronae while in other regions such hot material is absent. The boundary between these different regions in the HR diagram is indicated by several dividing lines based on different observational criteria (cf. Linsky \& Haisch 1979; Ayres et al. 1981; Haisch et al. 1991, 1992). Sensitive new ROSAT observations have substantially revised this picture. While Hünsch et al. (1996a) have shown from a complete, volume-limited sample that probably all but the very latest giants are X-ray sources of mostly about solar level, Reimers et al. (1996) detected X-ray emission from several bright giants and supergiants (the so-called hybrid stars) obviously violating the strict dividing line concept. Recently, Hünsch \& Schröder (1996) suggested a scenario in which the observed coronal properties of late-type giants and supergiants can be qualitatively understood in the context of stellar and angular momentum evolution.

It is obvious that all such investigations are severely hampered by the scarcity of X-ray detected evolved stars. A compilation of the Einstein detections is given by Maggio et al. (1990) and includes 69 detected giants and supergiants of spectral types F to M. Additionally, for 310 such stars upper limits of X-ray emission were derived. However, we have to keep in mind that the Einstein observations only covered about $10 \%$ of the sky with rather inhomogeneous exposure times. The Maggio et al. data consist of both pointed observations on individual stars and serendipitious observations in the field-of-view of other targets.

Contrary to the Einstein observations, the ROSAT observatory scanned the whole sky during the first half year of its mission. The ROSAT all-sky survey (RASS) resulted 
in about 150000 detected X-ray sources, which form a flux-limited but otherwise unbiased sample (Voges et al. 1996a).

We have searched for X-ray detections of optically bright late-type giants and supergiants in the RASS data. The aim of this paper is to provide the X-ray data of those detections in the form of a homogeneous catalogue. We describe the way the catalogue was created and how the $\mathrm{X}$-ray properties of the stars were derived. For a detailed astrophysical discussion we refer to an accompanying paper in the main journal (Hünsch \& Schmitt 1998).

We further note that a similar catalogue of all RASSdetected bright OB-type stars (irrespective of luminosity class) has already been published by Berghöfer et al. (1996).

\section{RASS data and detection of late-type stars}

\subsection{The ROSAT all-sky survey (RASS)}

During the first half year, the ROSAT observatory carried out the first all-sky survey with an imaging X-ray telescope. The whole sky was scanned along great circles perpendicular to the direction to the Sun. Because of the Earth's motion around the Sun, the plane of these circles slowly rotated around an axis through the ecliptic poles, thus covering the whole sphere within 6 months. Each point of the sky was observed several times as the scan paths of 2 degrees width (i.e., the field of view of the PSPC detector) progressed along the ecliptic. Therefore, the data of any particular source consist of a number of "snapshots" of up to $30 \mathrm{~s}$ duration, separated by the orbital period of the satellite $(\approx 90 \mathrm{~min})$ and distributed over an interval of at least 2 days. Towards the ecliptic poles, the cumulative exposure time increases due to the larger number of scans covering a particular position. Depending on the ecliptic latitude (and down-time due to radiation belts of the Earth), the effective exposure time varies between $\sim 100 \mathrm{~s}$ and $\sim 40000 \mathrm{~s}$ (at the poles), with typical values of $\sim 400 \mathrm{~s}$ on the ecliptic. Given a typical energy-conversion factor for soft sources of $610^{-12} \mathrm{erg} \mathrm{cts}^{-1} \mathrm{~cm}^{-2}$ (cf. Sect. 2.4) the typical detection limit of RASS observations (i.e., $\approx 0.015 \mathrm{cts} \mathrm{s}^{-1}$ ) amounts to $f_{\mathrm{x}} \approx 10^{-13} \mathrm{erg} \mathrm{cm}^{-2} \mathrm{~s}^{-1}$. For a more detailed description of the RASS we refer to Voges (1992) and Belloni et al. (1994). Details of the ROSAT observatory in general can be found in Trümper (1983) and Trümper et al. (1991), the PSPC detector used during the RASS is described by Pfeffermann et al. (1986).

The source detection was performed by means of a maximum likelihood algorithm (Cruddace et al. 1988) in the course of the standard analysis software system (SASS; Voges et al. 1992). The significance of an X-ray source is expressed by the likelihood $L i=-\ln (1-P)$, where $P$ is the probability of existence; e.g., a likelihood of $L i=7$ corresponds to a source existence likelihood of $99.9 \%$. The result of the SASS is a comprehensive list of several $10^{4}$ sources, each source described by the sky position in RA and Dec., its source detection likelihood, count rate, hardness ratio, extent, and corresponding errors. The data for the brighter X-ray sources have recently been released as the ROSAT All-sky Survey Bright Source Catalogue (Voges et al. 1996b), which contains sources with Likelihood $\geq 15$ and count rate larger than $0.05 \mathrm{~s}^{-1}$, that have at least 15 photons.

\subsection{Selection of stars}

We used the Bright Star Catalogue (BSC; Hoffleit \& Warren 1991) as input sample for our search of X-ray bright late-type giants. Specifically, we selected the stars according to their spectral classification, i.e., all stars of spectral types A, F, G, K, M, and C and luminosity classes I, II, III or intermediate classes (including III-IV). We further included those stars without MK spectral classification but with the old Harvard prefix "g", and we also included all composite-spectrum stars with at least one component being of the abovementioned kind. Since the BSC is complete down to apparent visual magnitude $\sim 6.2$ and has a rather sharp cut-off at $V \approx 6.5 \ldots 7.0$, the input sample forms a well defined (optically) flux-limited sample of stars. Given that stars of luminosity class III (or brighter) are normally absolutely brighter than $M_{V} \approx+1.5$, nearly all giants within a space volume of about $100 \mathrm{pc}$ radius around the Sun are included in the BSC. In total, our input sample contains 3839 stars.

Since about 1 percent of the sky was not included by the RASS, a few BSC stars are not covered by X-ray observations. Those stars with less than $50 \mathrm{~s}$ exposure time are listed in Table 1.

\subsection{Matches between input stars and RASS-sources}

Our aim was to find X-ray sources detected in the RASS close to the positions of the input sample stars defined in the previous subsection. In the first step, we extracted all $\mathrm{X}$-ray sources with a likelihood of $\geq 7$ in a square box of $4 \times 4$ arcminutes dimension centered on the position of any (i.e., 9110) BSC stars. To find out up to what distance from the optically selected input positions an X-ray source can reliably attributed to a star, we proceeded as follows: We first constructed a sample of 10000 random sky positions by means of a Monte Carlo simulation. That sample was subjected to the same match procedure as the 9110 BSC positions. The two-dimensional distribution of the offsets in right ascension and declination between optical and X-ray positions is shown in Fig. 1 for both samples. The corresponding histogram of the offsets (in arcseconds) is plotted in Fig. 2, where the number of matches in each distance interval is normalized to the corresponding annular area, and where a correction factor due to the different numbers of input positions (i.e., 9110 vs. 10000) 
Table 1. Stars of the input sample with less than $50 \mathrm{~s}$ exposure time or which are located in the region of the X-ray bright Vela supernova remnant

\begin{tabular}{|c|c|c|c|}
\hline $\mathrm{HR}$ & Name & Sp. type & $V$ \\
\hline 276 & & G8III & 6.32 \\
\hline 437 & $\eta \mathrm{Psc}$ & G7IIIa & 3.62 \\
\hline 527 & & G9III & 5.91 \\
\hline 731 & 27 Ari & G5III-IV & 6.23 \\
\hline 904 & $7 \mathrm{Eri}$ & M1III & 6.11 \\
\hline 935 & & M3III & 5.27 \\
\hline 959 & & K1III & 6.15 \\
\hline 1195 & & G9II-III & 4.17 \\
\hline 1285 & & KOIII & 6.59 \\
\hline 1316 & & K3-4III & 6.71 \\
\hline 2851 & $\eta \mathrm{CMi}$ & F0III & 5.25 \\
\hline 2864 & $6 \mathrm{CMi}$ & $\mathrm{K} 1+\mathrm{IIIBa} 0.4$ & 4.54 \\
\hline 2917 & & K2III & 6.76 \\
\hline 2955 & & G8III & 6.19 \\
\hline 2965 & & M2IIIab & 5.77 \\
\hline 2967 & & M3II-III & 5.56 \\
\hline 2974 & & G2 0 -Ia & 6.56 \\
\hline 2995 & & G6/8III & 6.89 \\
\hline 3095 & $1 \mathrm{Cnc}$ & K3+III & 5.78 \\
\hline 3376 & & KOIII & 6.28 \\
\hline 3390 & & K3III & 6.24 \\
\hline 3426 & & A6II & 4.14 \\
\hline 3444 & & MOIII & 5.71 \\
\hline 3445 & & F3Ia & 3.84 \\
\hline 3452 & & A5II & 4.77 \\
\hline 3461 & $\delta \mathrm{Cnc}$ & KOIII-IIIb & 3.94 \\
\hline 3477 & & G5III & 4.07 \\
\hline 3487 & & A1III & 3.91 \\
\hline 3496 & & F2Iab & 5.75 \\
\hline 3520 & & A2III & 4.93 \\
\hline 3534 & & $\mathrm{G} 8 \mathrm{III}+\mathrm{A} 3-5 \mathrm{~V}$ & 6.42 \\
\hline 3978 & & K1III & 6.52 \\
\hline 4007 & & M3III & 6.40 \\
\hline 4128 & & M2IIIe & 6.43 \\
\hline 4538 & & G6Ib & 4.97 \\
\hline 4704 & $\zeta^{1}$ Mus & K0III & 5.74 \\
\hline 4972 & & A8II-III & 6.33 \\
\hline 5122 & & K1III & 6.42 \\
\hline 5124 & & G5Ib & 6.01 \\
\hline 5251 & & G8-K0III & 5.91 \\
\hline 5362 & & G8III & 5.56 \\
\hline 5419 & & K1III & 5.97 \\
\hline 6076 & & K5III & 6.29 \\
\hline 6078 & & K4III & 5.94 \\
\hline 7069 & $111 \mathrm{Her}$ & A5III & 4.36 \\
\hline 7203 & & G6III & 6.05 \\
\hline 7217 & $o \mathrm{Sgr}$ & G9IIIb & 3.77 \\
\hline 7370 & & K4III & 5.69 \\
\hline 7388 & & K0-1III & 6.13 \\
\hline 7434 & & KOIII & 6.18 \\
\hline 7536 & $\delta$ Sge & $\mathrm{M} 2 \mathrm{II}+\mathrm{A} 0 \mathrm{~V}$ & 3.82 \\
\hline 7635 & $\gamma$ Sge & M0-III & 3.47 \\
\hline 7811 & & G6III & 5.66 \\
\hline 7813 & & KOIII & 6.41 \\
\hline 7886 & & M6III & 6.25 \\
\hline 7903 & & A0III & 6.08 \\
\hline 7913 & $\beta \mathrm{Pav}$ & A7III & 3.42 \\
\hline 7934 & $\sigma \mathrm{Pav}$ & KOIII & 5.41 \\
\hline 7941 & & M5II-III & 6.38 \\
\hline 7968 & $\iota$ Ind & K1II-III & 5.05 \\
\hline 8401 & $30 \mathrm{Aqr}$ & KOIII & 5.54 \\
\hline 8618 & 40 Peg & G8II & 5.82 \\
\hline 8660 & 45 Peg & gG6 & 6.25 \\
\hline 8929 & & K1III & 6.02 \\
\hline 9036 & $\varphi \mathrm{Peg}$ & M2.5IIIb & 5.08 \\
\hline
\end{tabular}
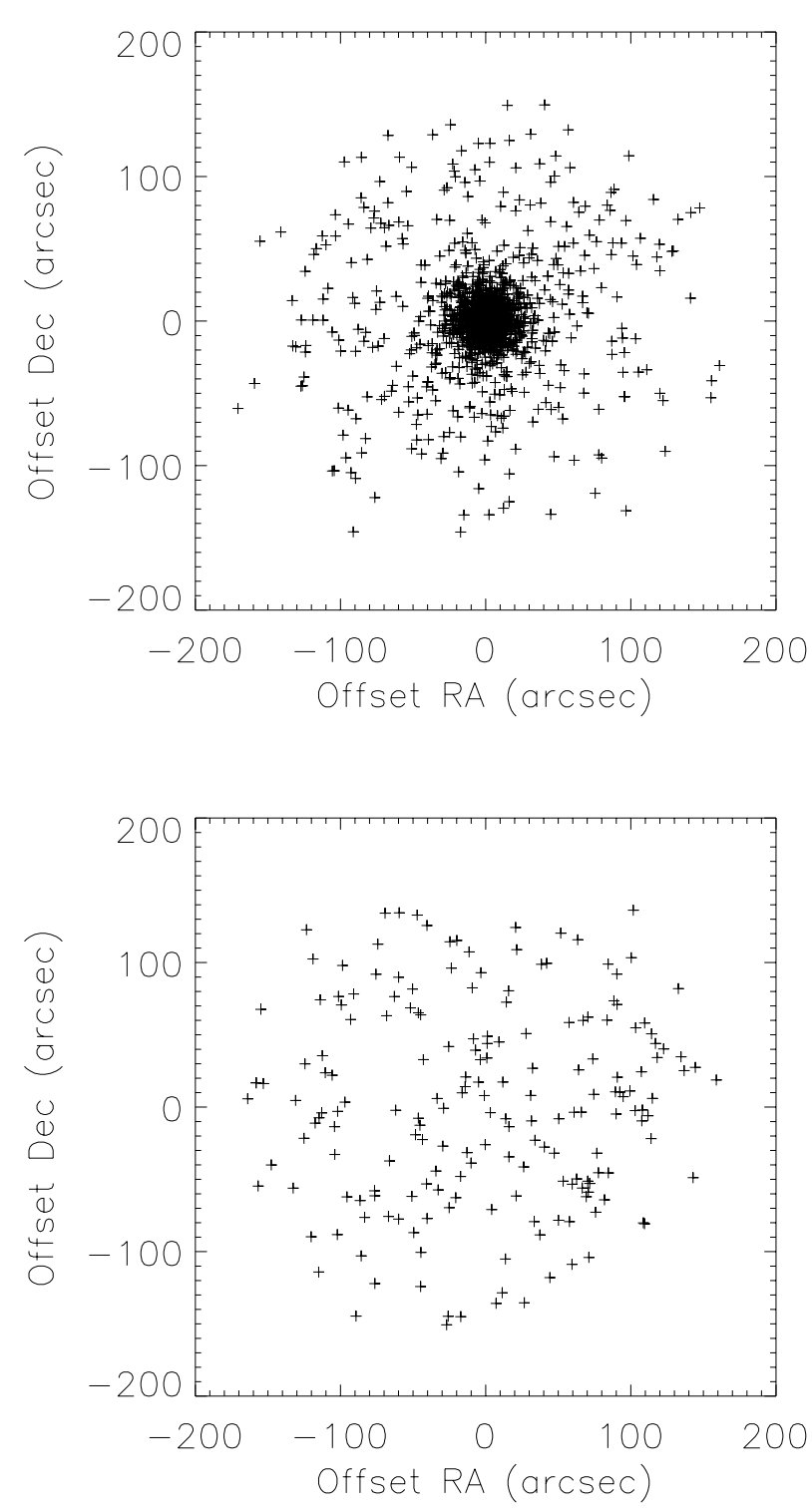

Fig. 1. Distribution of offsets in RA and Dec between optical and X-ray position for matches of RASS sources with the whole BSC star sample (top) and with 10000 random sky positions (bottom)

was applied. It is evident that for the BSC input sample the number of matches is strongly decreasing towards larger offset values, hence the vast majority of the obtained matches must be real. On the contrary, the similar number of matches for artificial sky positions is approximately independent of the offset distance (as expected), and has a more or less constant value of $\approx 2.6310^{-3}$ matches per square arcsecond. We further note that at an offset of $\approx 90$ arcseconds the number of artificial matches is about half the number of actual matches with BSC stars. That means, at that offset the (differential) probability of correctly identifying a BSC star with an X-ray source is about $50 \%$. 
Therefore, we chose 90 arcseconds as cut-off match distance between optical and X-ray position, up to which we attribute an X-ray source to a nearby BSC star.

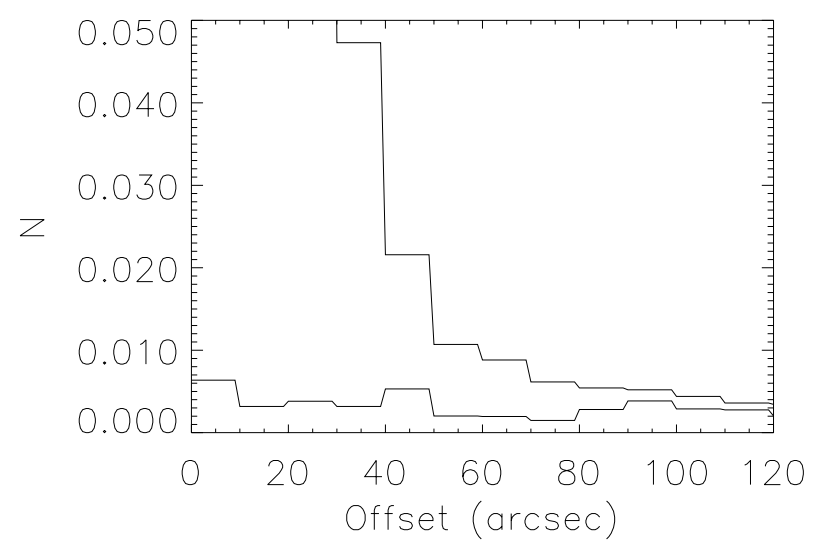

Fig. 2. Histogram of offsets for the BSC star sample (upper line) and the random positions (lower line). The $y$-axis is given in numbers of matches within a given offset interval, divided by the area of the corresponding annulus

For about 200 X-ray sources extracted in this way and not included in the Bright Source Catalogue (Voges et al. 1996b), we checked the X-ray images by eye for reality. Specifically, we rejected photon distributions that are significantly contaminated by nearby strong sources or that are obviously extended. In questionable cases, we ran the standard source detection algorithm of EXSAS on the source images in different passbands and decided on the basis of the results which sources to retain in our final catalogue.

Confining now attention to the 3839 BSC positions identified with late-type giants and supergiants, we detected X-ray emission from 450 stars, i.e., the average detection rate is $11.7 \%$. Since the total search area around these 3839 stars is $3839 \cdot \pi \cdot\left(1.5^{\prime}\right)^{2}=7.54 \square^{\circ}=0.018 \%$ of the sphere, and the total number of RASS sources amounts to $\sim 150000$, we would expect 27.4 chance coincidences of late-type giants or supergiants with background (or foreground) X-ray sources (i.e., $6 \%$ of our detected sources). If we consider a maximum offset of 60 arcseconds, this would be even reduced to 12.2 chance coincidences. At least for one case, HR 4289, this has been verified (Hünsch et al. 1996b).

\subsection{Determination of X-ray fluxes}

The conversion of count rates $(\mathrm{CR})$ into X-ray fluxes is generally performed by application of an energyconversion-factor (ECF):

$f_{\mathrm{x}}=\mathrm{ECF} \cdot \mathrm{CR}$.
ECF depends on both the underlying X-ray spectrum of the source and the amount of interstellar hydrogen absorption. As the source spectrum can be assumed to be thermal emission from an optically thin plasma in the case of late-type stars, the main determining parameter is the temperature of the plasma. However, in addition to the general problems of determining spectral parameters from low-energy-resolution proportional counter measurements, the detailed analysis of X-ray spectra requires quite a large number of photons, i.e., bright sources or long exposure times. For most of the sources in our catalogue, these requirements are not fulfilled, thus their coronal temperatures are unknown. Moreover, information on the interstellar hydrogen column density is also lacking for most of the stars. For late-type stars, colour excesses are difficult to measure due to the large intrinsic scatter of the colour indices. On the other hand, giants are intrinsically bright objects, on average they are far away and the amount of interstellar absorption cannot be neglected in most cases.

Fortunately, a rough information on the energy distribution in the X-ray range is provided by the hardness ratio.

$h r=\frac{\mathrm{H}-\mathrm{S}}{\mathrm{H}+\mathrm{S}}$,

where $\mathrm{H}$ and $\mathrm{S}$ denote the source counts in the hard $(0.5-2.0 \mathrm{keV})$ and soft $(0.1-0.4 \mathrm{keV})$ passbands of ROSAT. The hardness ratio is an "X-ray colour" that is influenced by both the plasma temperature and the hydrogen column density. Hünsch et al. (1996a) analyzed several PSPC pulse-height spectra of nearby giants and find from modelling the observed energy distributions by isothermal or two-temperature-component RaymondSmith (1977) models a linear relation

$\mathrm{ECF}=(5.30 \cdot h r+8.7) \quad 10^{-12} \mathrm{erg} \mathrm{cm}^{-2} \mathrm{cts}^{-1}$.

Note that Schmitt et al. (1995) find a very similar relation for late-type main-sequence stars.

Since the SASS source detection was separately performed in both passbands, and since most of our X-ray sources were detected in both bands, the hardness ratios can be determined for most stars, although in some cases with quite large errors. In a few cases, where the sources were not detected in the soft passband, we set $h r=+1.0$ by definition. The error in the hardness ratio is given by

$\sigma_{h r}=2(\mathrm{~S}+\mathrm{H})^{-1.5} \cdot \sqrt{\mathrm{S} \cdot \mathrm{H}}$.

From the hardness ratios, we calculated individual energyconversion-factors, which cover a range of ECF = $4 \ldots .1410^{-12} \mathrm{erg} \mathrm{cts}^{-1} \mathrm{~cm}^{-2}$. We refrain from estimating individual errors for $f_{\mathrm{x}}$ since the error in ECF is very difficult to quantify. In general, we estimate the error to be within a factor of two for the weaker sources and less for the brighter sources. 
Since the apparent flux $f_{\mathrm{x}}$ depends on the distance, a more characteristic measure would be the X-ray luminosity $L_{\mathrm{x}}$. However, reliable distance measurements from parallaxes exist only for a minority of our sample stars. Spectroscopic parallaxes from luminosity classes are quite uncertain and absolute magnitudes from the WilsonBappu effect exist only for part of the stars brighter than $V \approx 5$. Therefore, we did not calculate individual X-ray luminosities. A distance-independent measure of the level of X-ray emission is the ratio of X-ray to bolometric flux. We calculated bolometric fluxes from the relation

$f_{\text {bol }}=2.710^{-5} \cdot 10^{-\frac{m_{\mathrm{bol}}}{2.5}} \quad \mathrm{erg} \mathrm{cm}^{-2} \mathrm{~s}^{-1}$,

where the apparent bolometric magnitude is given by $m_{\text {bol }}=V+$ B.C. The bolometric corrections B.C. were taken from the tables of Schmidt-Kaler (1982) as given in the Landolt-Börnstein, by interpolating the values in colour index $B-V$ and luminosity class whenever necessary. For the M-type stars, we used the spectral type instead of the colour index due to the weak dependence of B.C. on $B-V$.

\section{The catalogue}

The table contains optical and X-ray data of all 450 detected late-type giants and supergiants including A-type stars listed in the BSC.

The columns of the table contain the following information:

Column 1: HR number (BSC)

Column 2: HD number

Column 3: the star's name (Bayer or Flamsteed designation)

Column 4: $V$ magnitude (from BSC)

Column 5: $B-V$ colour index (from BSC)

Column 6: MK spectral classification (from BSC)

Column 7: binary flag; S: single star, VB: visual binary (if a companion within 90 arcseconds distance is known), SB: spectroscopic binary (as given in the BSC), RS: RS CVn system (usually also SB), B: other binary (composite spectrum), CV: cataclysmic companion (HR $4765=4$ Dra), Symb: symbiotic star (HR $8992=\mathrm{R}$ Aqr)

Column 8: effective exposure time in seconds

Column 9: mean PSPC count rate in counts per second Column 10: error of PSPC count rate

Column 11: likelihood of existence (cf. Sect. 2.1)

Column 12: offset in arcseconds between optical and X-ray position

Column 13: hardness ratio $h r=(\mathrm{H}-\mathrm{S}) /(\mathrm{H}+\mathrm{S})$ (cf. Sect. 2.4)

Column 14: error of hardness ratio

Column 15: apparent X-ray flux $(0.1-2.4 \mathrm{keV})$ in
$10^{-14} \mathrm{erg} \mathrm{cm}^{-2} \mathrm{~s}^{-1}$ (see Sect. 2.4)

Column 16: logarithm of X-ray to bolometric flux ratio (see Sect. 2.4).

Acknowledgements. The ROSAT project is supported by the Bundesministerium für Bildung, Forschung und Technologie (BMBF/DARA) and the Max-Planck-Gesellschaft (MPG). We would like to thank our colleagues from the MPE ROSAT group, especially T. Berghöfer, for their support. MH acknowledges the hospitality of the MPE during his stay in Garching.

\section{References}

Ayres T.R., Linsky J.L., Vaiana G.S., Golub L., Rosner R., 1981, ApJ 250, 293

Belloni T., Hasinger G., Izzo C., 1994, A\&A 283, 1037

Berghöfer T.W., Schmitt J.H.M.M., Cassinelli J.P., 1996, A\&AS 118, 481

Cruddace R.G., Hasinger G.R., Schmitt J.H.M.M., 1988, The application of a maximum likelihood analysis to detection of sources in the ROSAT data, in: Murtagh F., Heck A. (eds.) Astronomy from Large Databases, ESO Conf. Workshop Proc. 28, 177

Haisch B.M., Schmitt J.H.M.M., Rosso C., 1991, ApJ 383, L15

Haisch B.M., Schmitt J.H.M.M., Fabian A.C., 1992, Nat 360, 239

Hoffleit D,E., Warren W.H.jr., 1991, The Bright Star Catalogue, 5th Rev. Ed., Yale Univ. Obs., New Haven

Hünsch M., Schmitt J.H.M.M., 1998, A\&A (in preparation)

Hünsch M., Schröder K.-P., 1996, A\&A 309, L51

Hünsch M., Schmitt J.H.M.M., Schröder K.-P., Reimers D., 1996a, A\&A 310, 801

Hünsch M., Reimers D., Schmitt J.H.M.M., 1996b, A\&A 313, 755

Linsky J.L., Haisch B.M., 1979, ApJ 229, L27

Maggio A., Vaiana G.S., Haisch B.M., et al., 1990, ApJ 348, 253

Pallavicini R., Golub L., Rosner R., et al., 1981, ApJ 248, 279

Pfeffermann E., Briel U.G., Hippmann U., et al., 1986, Proc. SPIE 733,519

Raymond J., Smith B.W., 1977, ApJS 35, 419

Reimers D., Hünsch M., Schmitt J.H.M.M., Toussaint F., 1996, A\&A 310,813

Schmidt-Kaler T., 1982, in: Voigt H.H., Schaifers K. (eds.) Landolt-Börnstein VI, 2b. Springer, Heidelberg

Schmitt J.H.M.M., 1997, A\&A 318, 215

Schmitt J.H.M.M., Fleming T.A., Giampapa M.S., 1995, ApJ 450,392

Trümper J., 1983, Adv. Space Res. 2, 241

Trümper J., et al., 1991, Nat 349, 579

Vaiana G.S., Cassinelli J.P., Fabbiano G., et al., 1981, ApJ 244,163

Voges W., 1992, in: Proc. of the ISY Conf. "Space Science", ESA ISY-3, ESA Publ., p. 9

Voges W., et al., 1992, in: Proc. of the ISY Conf. "Space Science", ESA ISY-3, ESA Publ., p. 223

Voges W., et al., 1996a, MPE Report 263, 637

Voges W., et al., 1996b, IAUC 6420 